\title{
Literature Review and Synthesis Implications on Healthcare Research, Practice, Policy, and Public Messaging
}

Susan W. Buchholz and Kirsten A. Dickins

Literature reviews and syntheses invaluably contribute to understanding the state-of-the-science for healthcare research, clinical practice, health policy, and public messaging.

\section{CHAPTER OUTLINE}

- Objectives

- The Multidisciplinary Value of Literature Review and Synthesis

- Implications of Literature Review and Synthesis on Healthcare Research

- Implications of Literature Review and Synthesis on Clinical Practice

- Implications of Literature Review and Synthesis on Health Policy

- Implications of Literature Review and Synthesis on Public Messaging

- Global Trends Impacting Literature Reviews and Syntheses

- Final Thoughts

- Activities

- Summary Points

- References

In this final chapter, we provide an overview of the multidisciplinary value of literature reviews and syntheses. Literature reviews and syntheses uniquely inform healthcare research, clinical practice, health policy, and media messaging for experts and the lay public. Literature reviews and syntheses can guide new understandings of the current literature and provide direction for future research. Additionally, they contribute to the knowledge databases and guidelines that clinicians use to inform practice decisions. Literature reviews and syntheses are also leveraged to accurately integrate existing 
information to inform relevant health policy decisions. There are different public venues that can be used to convey important knowledge gleaned from the work. Global trends continue to shape review processes and scale of impact. Once you have learned how to conduct a literature review and synthesis, you have gained a skill set that will undoubtedly serve you throughout your career.

\section{LEARNING OBJECTIVES}

In this chapter we discuss why literature reviews and syntheses are valuable across professional disciplines. By the end of this chapter, you will be able to:

- Describe how conducting a literature review and synthesis adds value across disciplines

- Understand the implications of literature review and synthesis on understanding and guiding future healthcare research endeavors

- Recognize the impact of literature review and synthesis on clinical practice decision-making

- Identify the influence of literature review and synthesis on health policy decision processes

- Determine opportunities and best practices for using literature reviews and synthesis in public messaging efforts

- Appraise global trends that influence literature review and synthesis guidelines and standards

- Summarize the integral components of the literature review and synthesis process and outcomes

\section{THE MULTIDISCIPLINARY VALUE OF LITERATURE REVIEW AND SYNTHESIS}

The existential question may occur to the reviewer sometime during the literature review and synthesis process: "Why does this matter?" Or "Why am I doing all of this work?" "Towards what end?" After all, a literature review and synthesis does require a great deal of time, focus, and energy. We would like to assure you of the host of varied reasons substantiating the value of the literature review and synthesis. One of the most obvious is the academic assignment-or that which is required of a student for a course that is part of an educational or training program. However, there is still an overarching objective informing the literature review assignment: Although it is a time-intensive endeavor for a student, it generally requires an investment of time and energy on behalf of the faculty member who is guiding the student, as well (particularly for the novice student reviewer). Literature reviews and syntheses invaluably contribute to understanding the state-of-the-science for healthcare research, clinical practice, health policy, and public messaging. Rigorously conducted literature reviews can be a powerful source of information for other researchers, regardless of the discipline. Literature 
reviews and syntheses provide researchers with the opportunity to see the state-of-thescience from an elevated and consolidated vantage point, making apparent where the gaps in the literature exist and providing the platform for determining focus. The reviewer and reader can become familiar not only with the precise topic being explored, but who the experts are on a given topic, popular methods employed, important findings, and recommended directions for future healthcare research. It is also possible that the reviewer may already be considered a topic expert and may even review their own work. With the knowledge gained from the literature review and synthesis process, a reviewer can decide to take their work in a variety of directions, now immersed in and informed by the literature regarding a question or topic.

There are nearly 8 billion people in the world (U.S. Census Bureau, 2021) and, throughout the world, there are healthcare scientists who diligently work to improve the health and healthcare for the citizens of individual countries and the global community. Every day, nurses and other healthcare professionals contribute knowledge to the global literature database, reporting on their studies and other scientific work. These studies are conducted and reported with respect to what is meaningful and useful for specific groups of people, accounting for cultural considerations and using this information to improve healthcare across settings (Oh, 2016). Internationally, many people have access to healthcare literature from varied resources, while significant issues persist with respect to equitable access to electronic databases and other relevant resources. Developing a diverse review team on which at least one team member has data access is one method to addressing this concern, albeit an imperfect one. Once nurses and other healthcare professionals distill resources that are most rigorous and reputable, there still remains an abundance of healthcare literature. There are multiple databases in multiple languages that house this literature. Libraries of universities and other institutions typically grant access to a fair number of these databases, through which reviewers can quickly access the literature. PubMed offers free access to all healthcare scientists with online access (National Library of Medicine, National Center for Biotechnology Information, 2021). Although not all articles are freely available via PubMed, the title and abstract can typically be accessed and viewed, so that the reader can ascertain an understanding of the article. In addition to the many databases, there are highly respected and long-established organizations that support the development and housing of rigorously completed literature reviews (e.g., Cochrane, Joanna Briggs Institute, and the Centre for Evidence-Based Medicine) that facilitate further access to information internationally.

The ease of article accessibility seems quite the distance away from a memory of the older of the two authors on this book. She remembers when studying for her dissertation work, she went to the library, checked for potentially relevant articles in large reference books, located the print copy of the articles stored on one of the many rows of large shelves, and printed it on the copier as she methodically inserted coins. Now that very same author (albeit a few years older) can sit down with her cup of tea and her laptop computer and complete an initial advanced search of the literature, download available articles, and order electronic copies by inter-library loan, all in a morning's work. She can also conduct a search of the grey literature on that same laptop, checking on what may have not yet 


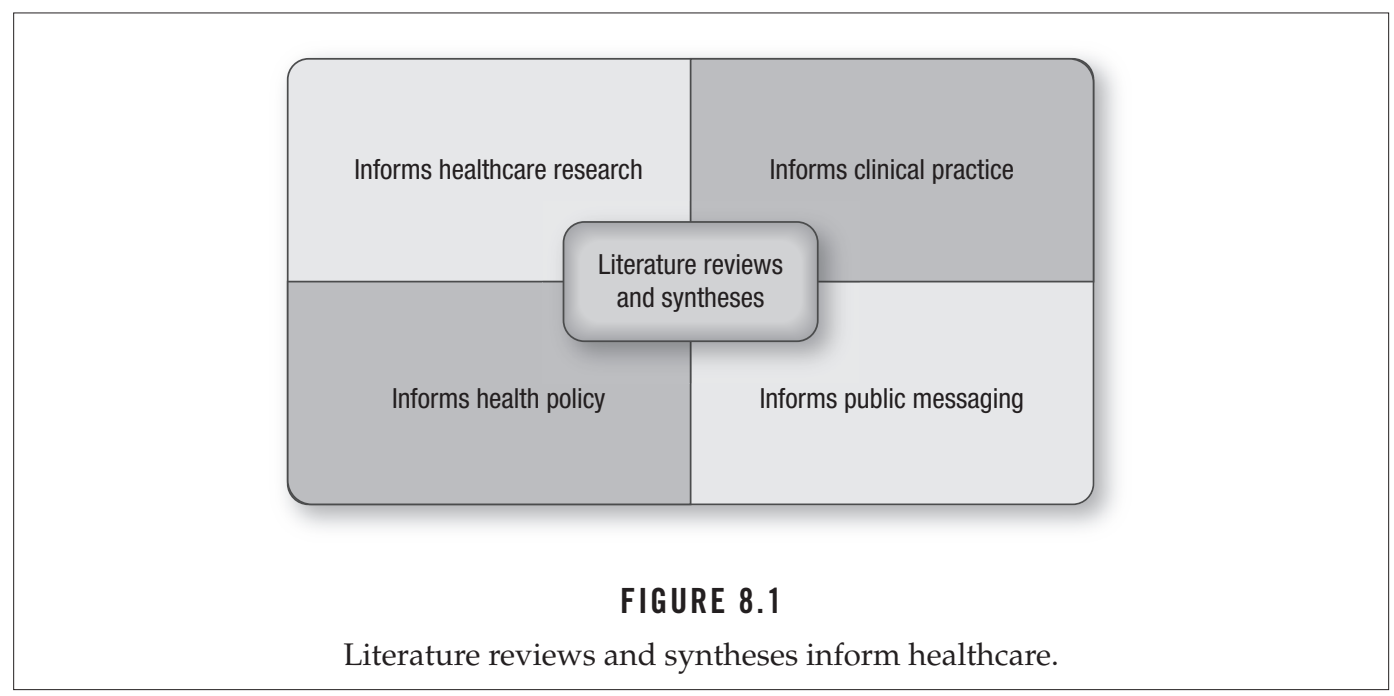

made its way to a formal database. However, being able to efficiently and effectively synthesize the literature requires the commitment of time, training, and skills, and the ability to locate the resources necessary for appraisal and interpretation (Oh, 2016). These are skills well worth learning and having, as literature reviews and syntheses invaluably contribute to understanding and informing healthcare as noted in Figure 8.1. These areas include healthcare research, clinical practice, health policy, and public messaging.

\section{IMPLICATIONS OF LITERATURE REVIEW AND SYNTHESIS ON HEALTHCARE RESEARCH}

Literature reviews and syntheses are important to the work of healthcare researchers. Reviews distinctly and comprehensively inform healthcare researchers of the methods, results, and implications of research completed on a given topic to date, as well as an understanding of the research gap. There are many benefits for the reviewer to fully appreciate the research that has and has not been undertaken. Having a solid understanding of who has conducted which studies, where the studies were conducted, which methods were employed, and key findings provides the reviewer and reader with both an overview and critical specifics about a given topic or question. Repeating studies that have already been conducted which do not require a timely update is not an optimal use of research resources. However, informing research with knowledge generated by prior literature, as well as evidence gaps in a particular context or with specific populations, can be a productive use of a researcher's time.

Awareness of evidence gaps helps to provide specific direction for future research efforts. Reading and understanding the synthesis of the discussion, implications, and future directions of multiple data sources can help determine the magnitude or consensus around recommended future research directions. While there is value in recognizing what is recommended in peer-reviewed, published literature, we caution that 
recommendations should be met with a Reviewer's creative innovation. It is useful to appreciate that evidence gaps comprise one aspect of informing future research. The reviewer is advised to combine recommendations for future research with their own expertise, interests, and methodological strengths. The experiences of a reviewer within their role shapes what they determine is necessary and practical for guiding future scholarly endeavors.

We recommend that the reviewer also search the grey literature, including literature with null results. In the event of null findings, it is important for researchers to submit their work for publication, as null findings represent a valuable contribution to the stateof-the-science (Nallamothu et al., 2020). Even if the reviewer can locate null findings in the scientific databases, it is still helpful for the reviewer to examine the grey literature, comprehensively painting a picture of the available evidence on a review topic or question.

\section{IMPLICATIONS OF LITERATURE REVIEW AND SYNTHESIS ON CLINICAL PRACTICE}

Healthcare clinicians utilize scientific findings to inform their clinical decision-making, in addition to expert opinion, patient preferences, and other resources. Practice guidelines and recommendations may potentially alter disease trajectory, health outcomes, and, relatedly, quality of life. Furthermore, patient, family, and community preferences, and social and structural determinants of health, will unquestionably shape the decisions regarding one's own health (Eden et al., 2011). Without considering the granularity of the meaning of a literature review to a patient, family, or community, the clinician may lose perspective when studying an area that has the potential to positively contribute at these levels.

The clinician may read the literature review synthesis from several different perspectives, informing how they may approach a patient when discussing the patient's health and healthcare decisions. When a question is sharply focused, a literature review - and most often a systematic review - can provide useful information, including an analysis and interpretation of information that can be used to inform clinical practice (Bolderston, 2008; Cronin et al., 2008). The clinician will assess the possible consequences of a health and healthcare management decision, the potential risks and benefits, and how these considerations are weighed or personalized to each individual patient and/or family. Clinicians may attempt to determine how reliable the evidence is before providing a recommendation. Regarding the evidence, the clinician will want to consider specific features of the patients for whom they are providing care. Literature reviews may provide additional guidance to address those patient-centered considerations (Eden et al., 2011). Examples include age, sex, race and ethnicity, socioeconomic status, or other clinical/diagnostic considerations. For example, with respect to population age, when a clinician is taking care of an infant, the clinician may seek out pediatric-specific literature reviews, in comparison to a healthcare clinician taking care of an individual who is 90 years old, seeking out geriatric-specific literature reviews. 
Clinicians often search first for a recent and rigorously completed systematic or other type of literature review on a given topic or question when building upon their current clinical knowledge base. If unable to locate a relevant literature review, they may decide to conduct their own literature review and synthesis (Khan et al., 2011). If time and resources are lacking to conduct their own review, then they may seek out the highestevidence level of individual studies to inform clinical decision-making.

Systematic reviews have become an integral component of clinical practice guidelines (Moher et al., 2015). When clinical practice guidelines are being created at the regional, national, or international level, it is typical that a comprehensive group of interdisciplinary professionals is gathered. These advisory groups use multiple sources of information to inform their discussion and guideline writing processes, including scientific evidence, their own expert knowledge, and, equally important, the values and preferences of the people whom the guidelines will serve. Scientific evidence includes rigorously completed systematic reviews and meta-analyses. This comprehensive and transparent process informs a solid process for optimizing the quality and outcomes of healthcare provision (Eden et al., 2011; Khan et al., 2011). Clinical practice guidelines help to decrease practice discrepancies and provide standardized guidance for the translation of often complex and vast research into practice settings (Institute of Medicine [IOM], 2011a). Importantly, clinical practice guidelines provide clinicians with the ability to assess benefits and harms in the decision-making process with each individual patient and family.

In contemporary healthcare contexts, clinicians must quickly sort through an expansive body of evidence both for relevancy and quality when making complex clinical decisions (Bettany-Saltikov \& McSherry, 2016; Eden et al., 2011). Of particular concern is when evidence is perceived to be biased or non-inclusive of a certain population, rendering it difficult for clinicians to determine how much weight to provide certain evidence. Clinicians should use an objective lens when viewing grey literature that may be industry-sponsored. In rigorous literature reviews and syntheses that include a critical appraisal/quality assessment process, the clinician may be able to use the quality rating to determine the level of the evidence. Critically appraising, assessing the quality, and synthesizing scientific evidence are foundational to clinical practice. As already noted, combining expert information from the literature needs to be coupled with both the judgment of the clinician, as well as the preferences of the patient receiving care (Boland et al., 2017). The increasing demand for evidence-based practice requires an increase in the number of clinicians who have the ability to both generate and synthesize evidence (Oh, 2016).

\section{IMPLICATIONS OF LITERATURE REVIEW AND SYNTHESIS ON HEALTH POLICY}

Literature reviews and syntheses are of central importance to health policy processes and decisions, often providing a concise summary of evidence applicable to health policy. For instance, providing information on the effectiveness of an intervention, the outcomes of 
a program, or an evaluation of the impact of a prior or current policy may serve as the evidence that informs a future health policy decision. When synthesizing the evidence within a literature review, with the professional judgment of a health policy maker, the input of constituents, and resources that are available to enact change, a reviewer can inform important decisions with comprehensive information that has a scientific backing (Boland et al., 2017). Health policy makers may choose to complete a literature review to provide information that will be relevant to a particular policy decision. A concentrated review process can help to address targeted policy questions relatively quickly. As with all literature review teams, health policy decision-makers need to have a team that includes the necessary expertise, skills, and experience to conduct a literature review.

Literature reviews that primarily or secondarily include a cost-effectiveness analysis can guide policy makers in the decision-making process, particularly in a setting with resource constraints (Booth et al., 2016). The actual and projected costs of a program must be informed by not only what the program offers, but also by the anticipated impact, including numbers of persons or communities, as well as predictions of select outcomes. Costs consist of many components, including money, time, and talent, as well as other resources-all of which are finite.

Policy-informing reviews can come in the form of a rapid review, meant to abbreviate the review process timeline to expedite and more quickly inform relevant policy decisions. Reviewers can facilitate the uptake of rapid reviews by developing partnerships with policy makers or health systems managers and providing education about the validity and applicability of rapid review results, as well as how to identify rapid reviews, assess, and interpret findings. While a systematic review is often considered the gold standard to inform a pertinent health policy decision, systematic reviews can be costly regarding time and resources. If there is a short turn-around time available for a health policy decision and a systematic review is not possible, a rapid review may be utilized. However, the reviewer should always follow a rigorous protocol (Grant \& Booth, 2009). Early and sustained engagement with the reviewer and the health policy stakeholders are essential for focusing the rapid review and ensuring that it is appropriate to a specific health policy decision of interest. While rapid review methods can be streamlined at many stages of the review process, the review must document and transparently report methodological choices to ensure that the evidence review fits the stated purpose and/or health policy decision. Although rapid reviews can be helpful for healthcare decision-making, policy makers and health systems managers need to consider the context of rapid reviews when using them to inform decision-making. While rapid reviews can inform a busy reader of pertinent issues and considerations, they are necessarily concise (Pautasso, 2013). Rapid reviews are typically shorter in length, and need to be focused to be maximally useful to the policy reader. The rapid review should provide easily and quickly comprehensible study results, emanating from clear and concise methods for reporting statistical analyses. Foci need to be placed on messaging to key audience members, examining results, and detailing policy implications (Tricco et al., 2017).

When rapidly reviewing the literature to inform a policy decision, a reviewer may find evidence risks outweigh any potential benefit. Or there may simply be insufficient evidence 
available to inform a particular decision. Be aware that actions on the part of individuals or political groups may minimize the findings of a review that offers opposing opinions (Booth et al., 2016). Political interest potentially adds another layer of complexity in the use of literature reviews and syntheses, requiring thoughtful actions by the health policy makers who are working to promote and use science for the betterment of public health.

Having an extensive, methodical review of the literature that is quickly and easily comprehensible is incredibly valuable in health policy realms. A quickly comprehensible review first includes the title and abstract, which is a consolidated overview of the literature review background, methods, results, and conclusions. A short plain-language summary can also provide accurate and easily interpretable information. Executive summaries provide more information than an abstract but are still precisely that: a summary, which should be quick to read and easy for the reader to efficiently comprehend the main points. Executive summaries are designed to display the main points of the literature review within a restricted or abbreviated page count. Further, it is often helpful to display the results of the literature review and synthesis in easily navigable tables. When methodologically appropriate, meta-analyses and meta-syntheses provide concise summarized evidence which can be used to inform policy decision-making. Infographics can be useful as an aesthetically diverse method to convey certain main points or specific sections of a literature review.

\section{IMPLICATIONS OF LITERATURE REVIEW AND SYNTHESIS ON PUBLIC MESSAGING}

Literature reviews are undoubtedly instructive in informing healthcare research, clinical practice, and health policy; equally important, however, is ensuring that the public has access and exposure to the knowledge generated by literature reviews in a critical and meaningful way. Researchers and reviewers often publish science in peer-reviewed journals and present at society and other academic conferences. Clinicians contribute to the creation of clinical practice guidelines and are informed by the literature reviews that provide the evidence for evidence-based decisions. Health policy makers leverage literature review approaches to aggregate the literature toward making a health policy decision, which may be implemented at a macro scale. However, when communicating the results of a literature review to the public, different strategies and venues must be considered for maximal public impact.

There is a rapidly expanding number of global media platforms, usable to disseminate the results of a literature review and synthesis to the public. Reviewers have many opportunities to inform the public of their work, such as interviewing with a public news organization, or writing an Op-Ed for a news outlet. Social media, including Twitter, Facebook, and other venues, are platforms that can be used to quickly disseminate literature review work to a large audience. Short video clips on platforms such as YouTube also facilitate the sharing of literature review findings. Additionally, reviewers can create websites or post pertinent information regarding their work on existing websites, such 
as an institutional research website (in which case the institution's website posting guidelines need to be carefully followed).

Formatting information for the public about your literature review and synthesis requires a distinct and different approach from that used in a peer-reviewed article. How a reviewer formats the findings of their literature review is largely dependent upon the target audience. Using language written at an appropriate literacy level is necessary to effectively deliver your message to the public. We cannot emphasize enough the importance of using language that is tailored to your audience, avoiding jargon and complex sentences. Providing short, succinct messages with key points (e.g., bullets) alongside understandable tables can maximize interpretability and therefore impact. Including a publication date is also important to convey to the reader when the literature review and synthesis was published, as well as including the timeframe of the included data sources. Including pictures, figures, infographics, as well as white space can create a layout that helps the reader more quickly grasp important and key content of the literature review. If needed, the review team can consult with marketing and public relations within their institution for guidance on writing for the public. This can help in ensuring that your messaging retains critical scientific accuracy while appealing to the lay audiences that you are intending to reach.

When you circulate your work in publicly accessible domains, it is essential that you are prepared for the potential positive and negative outcomes that comes with shining a public light on your work. When sharing your work publicly, you may receive interest and praise for your contributions, while still receiving criticism, regardless of your intended impact. This may be particularly true if your literature review and synthesis might reveal controversial findings. For example, your literature review and synthesis may find unexpected and harmful effects of a particular treatment or intervention. If this is the case, you may want to consider working with your public relations, legal team, and/or other experts at your institution to determine how to best convey your literature review and synthesis findings while maintaining strict accuracy on reporting of the results. Know that, in general, if the literature review and synthesis has been carefully and rigorously completed, you can be confident in your synthesis of the findings.

We would be remiss if we did not include a discussion regarding sharing your work with the spoken word, meaning in conversations, for example with your neighbor or someone else with questions whom you might encounter informally. People who are not immersed or familiar with your work may be quite intrigued (or not) with what you are doing and listen attentively (or not). You may encounter disagreement, in which your informal conversational partner may cite a lay article that they read online, a podcast that they recently heard, or a Facebook post that they happened upon which challenges or contradicts your work. This person may also have a relative or friend who had an experience that relates to and potentially challenges your findings. Others may provide examples that are singular and anecdotal and may not necessarily systematically synthesize scientifically derived results. It can be helpful to attempt to understand what is shaping that person's perspective, to listen attentively to any questions, and to assert and respond with objectivity and humility. In these cases, be prepared to provide a respectful, scientifically accurate, understandable, concise, and easily comprehensible 
response. When the opportunity presents itself, you can explain in lay terms your methods used to derive the review findings, emphasizing the aggregation and synthesis of multiple and rigorously determined scientific sources.

\section{GLOBAL TRENDS IMPACTING LITERATURE REVIEWS AND SYNTHESES}

There has been a recent exponential expansion of global access to the internet, diverse new outlets, and social media platforms. Even throughout the writing of this text, the number of media platforms has expanded, as have the options available to display and disseminate information. Media platforms are most relevant and tailored to different audiences, and used in different ways for different purposes by different populations. If in doubt about which media platform to use and how to use it, we recommend that you consult the public relations and/or marketing department at your institution. Always have a solid understanding of the policies that exist pertaining to scientific dissemination within your institution. Also be mindful of a virtual permanence, in that what you post and disseminate is accessible in both real time and for the indefinite future by audiences far and wide. Keep this anticipated permanence front of mind prior to disseminating information on a public media platform, but also do not let this deter scientific dissemination. No healthcare professional wants to see a year, or a decade, or more of work overturned in a moment of misconstrued media attention, while also balancing recognition of the centrality of dissemination to scientific progress.

An increasingly popular trend is that of microlearning. People are consuming and digesting information in partialized units, potentially requiring only 5 to 10 minutes. The trend toward microlearning requires that information be communicated clearly and succinctly. When you are disseminating your literature review findings, it may be surprisingly challenging to determine how to present the information in shorter, consolidated sections. Reviewing the requirements of platforms that you choose to use, and finding examples of similar work can be helpful in making a decision on how to present your work.

There is also the possibility of encountering more publicly challenged or critiqued scholarship. This can present both unique challenges and relatedly, opportunities, for the review team as they interpret, translate, and present their work. This is especially true when evidence on a certain topic or in a particular field is nascent and rapidly evolving as scientists discover more about an issue. A manifestation of this trend has become self-evident in the setting of the COVID-19 pandemic. The collective understanding of the virus and its anticipated global impact change rapidly, as scientists and other healthcare professionals work at lightning speed to understand how to best prevent, diagnose, treat, and vaccinate for the virus. The political climate has also considerably influenced the scientific process and dissemination of related information, undoubtedly influencing public knowledge of the virus.

Evidence and Gap Maps (EGMs) are another tool used to provide guidance on research priorities. While systematic reviews provide a synthesize of the evidence, EGMs provide a summary regarding the availability of evidence and the areas in which the evidence may be 
sparse or limited. The Campbell Collaboration provides information on developing EGMs (www.campbellcollaboration.org/evidence-gap-maps.html). EGMs are displayed graphically, typically containing a matrix comprised of intervention categories in the rows and the identified outcomes in the columns. EGMs should comply with rules, including following a protocol, a specific established search strategy, specific inclusion and exclusion criteria, and reporting of all studies that meet eligibility criteria. The United Nations Children's Emergency Fund (UNICEF) provides an exemplar for how to create and share EGMs (UNICEF Office of Research-Innocenti, 2021). For specific guidance on EGMs, we refer you to White and colleague's 2020 article on "Guidance for Producing a Campbell Evidence and Gap Map” (www.onlinelibrary.wiley.com/doi/full/10.1002/cl2.1125).

The increasing availability and expanding importance of "big data" also has implications for literature reviews and syntheses, especially on the global scene (Alsunaidi et al., 2021). Researchers can now seek out answers from larger sets of data by analyzing big data sets, as well as conducting meta-analyses (Silva-Fernández \& Carmona, 2019). The increasing use of common data elements, minimal data sets, and the same measures across multiple studies, increases the availability of data for researchers to review. These data sets include data points and information on populations that historically would have been difficult to access, now potentially made more easily available for review across borders. This allows for broader comparison, as well as a more nuanced review and understanding of specific populations and issues.

As interest in and the number of literature reviews conducted and published continues to grow, so do the typology of reviews available for reviewers to embark upon. As the number of completed and published literature reviews and syntheses grows, umbrella reviews are becoming increasingly of interest-an option for reviewers when systematic reviews or other types of literature reviews have been completed on a particular topic or question of interest. Mapping reviews serve as another example, aiming to provide a broad overview of individual areas within a certain subject area. By mapping what research has already been completed within a specified area, reading and visualizing this map can provide the reviewer with greater awareness of how to move forward to address a particular gap (Booth et al., 2016). Of note, some types of literature reviews that have not been explored in depth within this text are currently gaining in popularity. For example, desk reviews prompt searching the literature for secondary data sources within a certain area, identify key areas of importance, and apply this information in a reportable format in a subject area, potentially in public health (USAID, 2014). No matter the type of review, the reviewer should understand the methods and approach that provide an unbiased report, adhering to contemporary standards and guidelines for the conduct of the selected type of review.

\section{FINAL THOUGHTS}

Providing a conclusion for a book about literature reviews is a bit like fitting a genie in a bottle. There is ample existing information on literature reviews and syntheses, with many experts and organizations leading the way with the conduct and dissemination of exemplary work. To these people that are either working independently or within an 
organization that provides resources for literature review, we wish to sincerely thank you for taking healthcare science to a level that historically seemed unimaginable. For you, the reviewer, simplistically speaking, there are four key things to remember with your own literature review and synthesis. First, you need to start. Second, you need to work persistently and steadily. Third, you need to finish. Fourth, you need to disseminate your literature review and synthesis contribution to ensure maximal impact. Take time to think about how you will disseminate to the right audiences, and that your literature review and the presentation thereof is sensical and intuitive for your target audience(s). It is also important to keep in mind that literature reviews are a part of the much larger landscape of research and evidence-based practice. Literature reviews and syntheses represent a distinct method poised to provide succinct information that can be used to guide healthcare research, clinical practice, health policy, and public understanding via appropriate messaging. However, literature reviews are still only one component of that process; other scientific evidence, clinical expertise alongside preferences, cultural context, and resources of the people impacted, must also factor strongly into the evidence base and related decisions that directly impact patients, families, and communities. Once you have completed your literature review and synthesis, you will have gained a skill set, and moreover, a way of thinking that will continue to serve you well throughout your career. We encourage you to continue reading literature reviews, periodically update your training in the area of literature reviews and syntheses, commit to finishing the work that you have begun, and always be a critical thinker who is thankful and gracious in the face of the critique that you will receive along the way. We also hope that you celebrate the work that you have done, and recognize and honor the value of the team members that have worked with you to ensure the successful completion of your literature review and synthesis.

\section{ACTIVITIES}

\section{Activity I}

As a content expert, you have been asked to produce a literature review and synthesis for a report that will be used in a legislative session. You are told that the review must be conducted and the report completed within one month. Fortunately, you are able to clear much of your calendar to engage in this task, as it has been deemed a priority by your institution. Consider how you would answer the following questions.

I. Identify a topic or question on which you identify as an expert.

2. With this topic and question in mind, think about what type of literature review would be a best fit for the topic and objectives, given your one-month timeline.

3. Try to identify potential review team members and justify each of their distinct roles on the team.

4. Create a list of process steps and timeline to follow to complete the literature review by the deadline.

5. Jot down notes on accountability measures to ensure methodological rigor in your review, especially considering the short turnaround time. 


\section{Activity II}

A recent literature review that you have written has immediate implications for a specific population in your locality. You decide to write an Op-Ed concerning the findings of the review to submit to one of the leading newspapers in your area. Think about how you may approach the Op-Ed writing process, including determining your unique angle. Toward this end, review the following websites for information on writing an Op-Ed.Then, answer the following questions.

www.theopedproject.org/oped-basics

www.commskit.duke.edu/writing-media/writing-effective-op-eds www.nytimes.com/2017 /08/25/opinion/tips-for-aspiring-op-ed-writers.html

I. What are the pros and cons of writing an Op-Ed by yourself or with others? If you decide to write with another person or people, who might you ask to help you to write the Op-Ed?

2. What is your proposed title? Leading line?

3. What elements of your review may be most enticing to highlight?

4. To whom would you alternatively submit your Op-Ed in the event that the intended newspaper decided not to publish it?

5. If you receive a call or message from a colleague or neighbor in response to your Op-Ed, how will you respond to their agreement or disagreement about what you wrote?

\section{SUMMARY POINTS}

In this text we have provided for you the necessary steps to conducting your own literature review and synthesis. In this chapter we provide a summary of the reasons justifying the time and cognitive energy investment needed in order to be successful in this work.

- Globally, there are a number of nurses and other healthcare professionals who conduct different types of research. These studies are published in peer-reviewed journals and may be available in the grey literature. This proliferation and availability of literature has in many ways prompted the growing number of literature reviews that are used to guide healthcare research, clinical practice, and health policy, as well as to inform public messaging on a topic.

- Literature reviews and syntheses provide healthcare researchers with rigorous information about work that has already been completed on certain topics, where the evidence gaps exist, as well as implications for future research. Reviewers conduct searches from health-related databases, as well as the grey literature, in order to conduct comprehensive reviews.

- Clinicians use the findings of individual studies and when available, literature reviews, along with their own expertise and patient values and preferences, to make evidence-based clinical decisions. Literature reviews, at a more macro level, are also often used to inform the development and modification of clinical practice guidelines. 
When needed, clinicians may also choose to conduct their own literature review and synthesis.

- Health policy decision-makers use literature reviews and syntheses to inform pertinent policy considerations, including cost-effectiveness, as well as risks versus benefits of a particular treatment or intervention. Health policy reviewers may choose to conduct rapid reviews to ensure that science informs policy decisions in a timely manner. Reviewers can also provide executive summaries of their literature reviews for efficient reading and integration into policy decisions.

- The lay public can benefit from literature reviews. Gearing literature review findings toward the public is of the utmost importance, ensuring dissemination and use by diverse audiences. There are several media platforms on which reviewers may choose to share their work. It is important to engage these media platforms wisely to ensure that the message is targeted to the appropriate audience, sometimes verifying regulations and recommendations with a public relations department. It is also advised to engage thoughtfully in conversations with others in a manner that promotes objectivity and humility in conveying literature review findings.

- The exponential expansion of global access to the internet continues to shape the impact of literature reviews. Important trends include microlearning, public challenges of expert recommendations, Evidence and Gap Maps (EGMs), "big data," and the multitude of literature review strategies that continue to influence the conduct and potential of literature reviews and syntheses.

- Exemplary work has been and currently is being completed in the global literature review and synthesis scene. When a reviewer chooses to undertake the literature review process, the reviewer needs to develop a plan to successfully complete the review and disseminate the finished product in a timely manner. Literature reviews are an integral component of the overall database of scientific knowledge that informs healthcare decisions. Once the reviewer has developed the skills to conduct literature reviews, the reviewer has entered into a process where they will continue to learn more over time about literature review and synthesis - an invaluable skill to hold in contributing to the generation of high impact healthcare knowledge.

\section{REFERENCES}

Alsunaidi, S. J., Almuhaideb, A. M., Ibrahim, N. M., Shaikh, F. S., Alqudaihi, K. S., Alhaidari, F. A., Khan, I. U., Khan, I. U., Aslam, N., \& Alshahrani, M. S. (2021). Applications of big data analytics to control COVID-19 Pandemic. Sensors, 21(7), 2282. https://doi.org/10.3390/s2107228

Bettany-Saltikov, J., \& McSherry, R. (2016). How to do a systematic literature review in nursing: A step-by-step guide (2nd ed.). Open University Press.

Boland, A., Cherry, M. G., \& Dickson, R. (2017). Doing a systematic review: A student's guide (2nd ed.). Sage.

Bolderston, A. (2008). Writing an effective literature review. Journal of Medical Imaging and Radiation Sciences, 39(2), 86-92. https://doi.org/S1939-8654(08)00057-X

Booth, A., Sutton, A., \& Papaioannou, D. (2016). Systematic approaches to a successful literature review (2nd ed.). SAGE.

Cronin, P., Ryan, F., \& Coughlan, M. (2008). Undertaking a literature review: A step-by-step approach. British Journal of Nursing, 17(1), 38-43. https://doi.org/10.12968/bjon.2008.17.1.28059 
Eden, J., Levit, L., Berg, A., Morton, S., \& Committee on Standards for Systematic Reviews of Comparative Effectiveness Research, \& Institute of Medicine. (Eds.) (2011). Finding what works in health care: Standards for systematic review. The National Academies Press.

Grant, M. J., \& Booth, A. (2009). A typology of reviews: An analysis of 14 review types and associated methodologies. Health Information and Libraries Journal, 26(2), 91-108. https://doi.org/10.1111/j.1471-1842 .2009.00848.x

Institute of Medicine (US) Committee on Standards for Developing Trustworthy Clinical Practice Guidelines, Graham, R., Mancher, M., Miller Wolman, D., Greenfield, S., \& Steinberg, E. (Eds.). (2011). Clinical practice guidelines we can trust. National Academies Press.

Khan, K., Kunz, R., Kleijnin, J., \& Antes, G. (2011). Systematic reviews to support evidence-based medicine (2nd ed.). Hodder Arnold.

Moher, D., Shamseer, L., Clarke, M., Ghersi, D., Liberati, A., Petticrew, M, Shekelle, P., Stewart, L. A., \& PRISMAP Group. (2015). Preferred reporting items for systematic review and meta-analysis protocols (PRISMA-P) 2015 statement. Systematic Reviews, 4, 1-4053-4-1. https://doi.org/10.1186/2046-4053-4-1

Nallamothu, B. K., Schultz, J., \& Petty, S. (2020). True negatives: Disseminating research on null, inconclusive, and confirmatory findings in cardiovascular science. Circulation: Cardiovascular Quality and Outcomes, 13(10), 7448. https://doi.org/10.1161/CIRCOUTCOMES.120.007448

National Library of Medicine, National Center for Biotechnology Information. (2021). Pubmed.gov. https:// pubmed.ncbi.nlm.nih.gov/

Oh, E. G. (2016). Synthesizing quantitative evidence for evidence-based nursing: Systematic review. Asian Nursing Research, 10(2), 89-93. https://doi.org/10.1016/j.anr.2016.05.001

Pautasso, M. (2013). Ten simple rules for writing a literature review. PLoS Computational Biology, 9(7), e1003149. https://doi.org/10.1371/journal.pcbi.1003149

Silva-Fernández, L., \& Carmona, L. (2019). Meta-analysis in the era of big data. Clinical Rheumatology, 38, 2027-2028. https://doi.org/10.1007/s10067-019-04666-3

Tricco, A. C., Langlois, E. V., \& Straus, S. E. (Eds.) (2017). Rapid reviews to strengthen health policy and systems: A practical guide. World Health Organization. https://apps.who.int/iris/bitstream/handle/10665/258698/ 9789241512763-eng.pdf;jsessionid=9A9AED7961D0D17D819AF294F5B96218? sequence $=1$

UNICEF Office of Research - Innocenti. (2021). Evidence gap maps. https://www.unicef-irc.org/evidence-gap -maps

United States Census Bureau. (2021). U.S. and world population clock. https://www.census.gov/popclock/world

USAID. (2014). Step 1: Complete desk review. https://assessment-action.net/wp-content/uploads/2014/03/ Phase-2-LEARN-Overview.pdf 
